\title{
Novel immunotherapy strategies for treatment of neuroendocrine neoplasms
}

\author{
Taymeyah Al-Toubah ${ }^{1}$, Mauro Cives ${ }^{2}$, Jonathan Strosberg ${ }^{1}$ \\ ${ }^{1}$ Department of Gastrointestinal Oncology, Moffitt Cancer Center, Tampa, FL, USA; ${ }^{2}$ Department of Biomedical Sciences and Human Oncology, \\ University of Bari, Bari, Italy \\ Contributions: (I) Conception and design: All authors; (II) Administrative support: None; (III) Provision of study materials or patients: None; \\ (IV) Collection and assembly of data: All authors; (V) Data analysis and interpretation: All authors; (VI) Manuscript writing: All authors; (VII) Final \\ approval of manuscript: All authors. \\ Correspondence to: Dr. Jonathan Strosberg. Department of Gastrointestinal Oncology, H. Lee Moffitt Cancer Center and Research Institute, 12902 \\ Magnolia Drive, 33612 Tampa, FL, USA. Email: jonathan.strosberg@moffitt.org.
}

\begin{abstract}
Neuroendocrine tumors (NETs) and neuroendocrine carcinomas (NECs) are a heterogeneous family of neoplasms. Well-differentiated tumors are often slow growing and characterized by low tumor mutational burden. Poorly differentiated NECs are aggressive, with an increased mutational burden and higher propensity to express PD-L1. While the therapeutic landscape for neuroendocrine neoplasms (NENs) has evolved substantially over the past decade, immunotherapy has been unexplored in NENs until recently. Checkpoint inhibitors such as anti-PD-1 and anti-CTLA-4 agents, bi-specific tumor-targeting antibodies, and chimeric antigen receptor (CAR) T-cell therapy are examples of treatments that have demonstrated efficacy in other cancers and have recently been investigated in NENs. This review examines the immune landscape of NENs in detail, summarizes recent clinical study results, and discusses potential future directions for immunotherapy.
\end{abstract}

Keywords: Carcinoid tumors; immunotherapy; immune checkpoints; PD-L1; PD-1; CAR-T cells

Received: 09 September 2019; Accepted: 14 November 2019; Published: 05 October 2020

doi: $10.21037 / \operatorname{tgh} .2019 .12 .18$

View this article at: http://dx.doi.org/10.21037/tgh.2019.12.18

\section{Introduction}

Neuroendocrine tumors (NETs) and neuroendocrine carcinomas (NECs) are a diverse family of neoplasms that range in behavior from indolent to highly aggressive. Well-differentiated NETs frequently originate from enterochromaffin cells in the gastrointestinal tract and lungs. They are characterized clinically by a relatively slow growth rate (compared to most malignancies) and a propensity to produce hormones and vasoactive substances (1). The genetic landscape of well-differentiated NETs typically consists of mutations in genes such as MEN1 and $D A X X$, which are associated with chromatin remodeling $(2,3)$. Tumor mutational burden is relatively low, and microsatellite instability is extremely rare $(4,5)$. Poorlydifferentiated NECs are highly aggressive malignancies, typically categorized as small cell and large cell. Small cell lung carcinomas and Merkel cell cancers are variants of poorly differentiated NECs which fall outside of the scope of this article. The mutational landscape of poorly differentiated NECs is similar to that of nonneuroendocrine cancers, with mutations in $p 53$ and $R b 1$ predominating. Tumor mutational burden is generally higher than observed in well-differentiated NETs (5-7).

Clinical trials of immunotherapy have only recently been completed in neuroendocrine neoplasms (NENs). Although data have demonstrated a relatively limited role for PD-1 inhibitor monotherapy, other immunotherapeutic approaches may yield improved results. In this article, we summarize the preclinical data on the immune landscape of NETs and NECs, and review the key clinical trials conducted thus far. 


\section{The immune landscape of NENs}

Multiple immune cell types, including T cells, NK cells, mast cells, macrophages as well as dendritic cells infiltrate NENs of different origins and grades. However, despite tumor infiltration and possibly immune recognition, NENs are able to escape the host immune response and avoid immunosurveillance by exploiting multiple local and systemic resistance mechanisms including the deactivation of $\mathrm{T}$ cells, dysregulation of $\mathrm{T}$ regulatory (Treg) cells and the creation of an immunosuppressive cytokine milieu with tolerogenic properties.

\section{Lymphocyte infiltration-pNETs}

Lymphocyte infiltration is a frequent event in both gastroenteropancreatic (GEP) and bronchial NETs. In a series of 87 pancreatic NETs (pNETs), CD3 + T cell infiltration was reported in $68 \%$ of the tumors and was not associated with tumor grade or other clinicopathological variables. Among patients with intermediate-grade pNETs, low-density lymphocyte infiltration appeared to predict recurrence following tumor resection compared to high density infiltration (8). Conversely, in a recent study of 244 GEP-NETs, high levels of intratumor lymphocyte infiltration were described to be significantly associated with higher tumor grade and shorter survival (9). Consistently, in a multispectral imaging analysis comparing 47 lowgrade pNETs with 5 high-grade pNETs and pNECs, T cell infiltration increased with grade (10).

\section{Lymphocyte infiltration-small bowel NETs}

In a cohort of $102 \mathrm{G} 1 / \mathrm{G} 2$ primary small bowel NETs, an intratumor host immune response was reported in approximately two-thirds of tumors, with the extent of the lymphocyte infiltration being significantly higher in duodenal NETs as compared with jejunal or ileal NETs (11). Of note, ectopic lymph nodes with activated germinal centers were observed at the tumor edge in about onefifth of the cases. In another study of 62 patients with small bowel NETs, lymphoid aggregates were found in $27 \%$ of tumors, and infiltration of CD8+ T cells was described in the $97 \%$ of the samples (12). At present, the biological significance of tertiary lymphoid structures in NETs remains unclear. Intriguingly, T lymphocytes have been reported to specifically recognize NET cells. Indeed, the presence of $\mathrm{CD} 8+\mathrm{T}$ cells reactive against NET-associated antigens such as chromogranin A or tryptophan hydroxylase has been demonstrated in patients with midgut NET (13). More recently, $T$ cells reactive against tumor neoantigens have been recognized in the blood of patients with metastatic rectal NETs (14).

\section{Lymphocyte infiltration—lung NETs}

In pulmonary NETs, the density of CD8+ T lymphocyte infiltration seems to parallel the degree of tumor differentiation. Indeed, a moderate-to-high host immune response has been detected in $7 \%$ of well-differentiated lung NETs (carcinoid tumors) and $20 \%$ of small cell and large cell NECs (15). In a study of 159 low- and high-grade pulmonary NETs, a high density of CD8+ T cells has been shown to independently predict both overall survival (OS) and progression-free survival (PFS) (16). By contrast, no association has been recently demonstrated between host immune response and survival outcomes in a study of 168 patients with typical or atypical lung carcinoids (17). In a retrospective analysis of 95 large cell NECs of the lung, infiltration of CD8+ and CD4+ T cells was recorded in $55 \%$ and $80 \%$ of cases respectively. Tumor infiltration by CD4+ T cells independently predicted shorter relapse-free survival (18).

\section{Treg-driven immunosuppression}

The presence of immunosuppressive FoxP3 + Treg cells has been shown to be more abundant in high- versus low-grade pNETs, and independently predicts dismal prognosis $(8,19)$. Regardless of the density of tumor infiltration, circulating levels of Treg cells have been found to be significantly higher in patients with midgut NETs as compared with healthy subjects, and the lower proliferative capability of $\mathrm{T}$ cells derived from patients with midgut NETs has been ascribed to a Treg-driven suppression of systemic Th1promoting cytokines such as IL-12 and IL-1b (20).

NK cells have demonstrated impaired cytolytic activity in GEP-NETs. In particular, a deficient interferon (IFN)-a response has been observed in patients with midgut NETs, where NK cell activity could be restored by exogenous treatment with interferon (21). Moreover, an increased NK cell activity has been associated with tumor regression (22). Mast cells may have a prominent role in pNET progression. Evidence from a mouse model of pancreatic b-cell tumorigenesis suggests that tumor-infiltrating mast cells regulate neoangiogenesis and tumor expansion. 
In this context, pharmacological inhibition of mast cell degranulation has proved effective in inducing cancer regression in mice harboring islet-cell tumors $(23,24)$.

\section{Tumor-infiltrating macrophages and antigen presentation}

Evidence from murine models suggests that tumorinfiltrating macrophages contribute to both angiogenic switch and pNET progression (25). Consistently, the density of macrophage infiltration appears to be higher in poorly differentiated NECs than in well-differentiated NETs (26). Large series studies have shown that a dense macrophage infiltration predicts recurrence following surgery $(27,28)$.

Antigen presentation is potentially impaired in NETs. Carcinoid-specific soluble immune inhibitory factors have been shown to down-regulate both maturation and function of dendritic cells in bronchial NETs (29). In addition, in a study of 104 surgically resected pNETs, the expression of HLA class I molecules has been demonstrated to be defective in $70 \%$ of cases (28). In another study, the MHC molecule b2-microglobulin has been shown to be altered in 10/11 samples of pNETs (30).

\section{Immune checkpoint inbibition}

In recent years, multiple investigations have been carried out to characterize the expression of the immune checkpoint molecules programmed death-ligand 1 (PDL1) and programmed death-1 (PD-1) in NETs and NECs $(9,11,17,18,28,31-41)$. As shown in Table 1, both the expression of PD-L1 and the extent of tumor infiltration by PD-1 lymphocytes appear to be higher in highgrade or poorly differentiated neoplasms rather than in well-differentiated tumors. Differences in the clinical characteristics of accrued patients (i.e., primary site, grade, fraction of metastatic cases), in the type of samples analyzed, in the mAb clone used for PD-L1 testing as well as in the criteria used for staining interpretation may account, at least in part, for the heterogeneity of results seen across different studies. It is currently unclear whether the expression of PD-1 or PD-L1 has any prognostic potential.

\section{Clinical trials of immune checkpoint inhibitors}

Several phase II studies have recently explored singleagent and combination therapy with immune checkpoint inhibitors.

\section{Monotherapy trials}

The KEYNOTE-028 study, a large multi-cohort phase $1 \mathrm{~b}$ study evaluating the safety and efficacy of pembrolizumab in patients with PD-L1-positive advanced solid tumors included 41 NET patients (42). Four (10\%) patients experienced objective responses while $71 \%$ experienced stable disease. Duration of response ranged from 6.917.6 months in the 4 responders. This data led to the inclusion of a NET cohort on the subsequent KEYNOTE-158 study. The KEYNOTE-158 study included a large cohort of "well and moderately differentiated" NETs originating in the lung, appendix, small intestine, colon, rectum, or pancreas (43). Patients were required to have progressed on at least one prior line of therapy, with no limit on the number of prior lines. Therapy consisted of pembrolizumab at a standard dose of $200 \mathrm{mg}$ every 3 weeks, for up to 2 years. The primary endpoint was overall response rate (ORR), assessed per RECIST 1.1 by independent central radiology review. Of the 107 patients who were treated on the NET cohort, $67.3 \%$ had received $\geq 2$ prior therapies and $15.9 \%$ had PD-L1 positive tumors [defined as combined positive score (CPS) $\geq 1$ on IHC analysis]. At the time of data cutoff, ORR was $3.7 \%$ (95\% CI, 1.0-9.3\%), with 4 partial responses (PR) and no complete response (CR). Of the four patients with PRs, three had pancreatic, and 1 had a gastrointestinal NET of unknown primary, all of whom had PD-L1 negative tumors. PFS was 4.1 months (95\% CI, 3.55.4 months) and the 6-month PFS rate was $38.2 \%$. Median overall survival (OS) was not reached at the time of data cut-off, and the 6-month OS rate was $84.6 \%$. Treatmentrelated adverse events (AEs) occurred in $75.7 \%$ of patients, with $20.6 \%$ having grade 3-4 AEs.

Another phase II study of pembrolizumab was conducted in high-grade NENs, excluding NENs of thymic or lung origin, who had progressed on prior platinum-based therapy (44). Therapy consisted of pembrolizumab at a standard dose of $200 \mathrm{mg}$ every 3 weeks, for up to 2 years. The primary endpoint was ORR per RECIST 1.1. Of the 21 patients enrolled, 15 patients had available archival tissue for PD-L1 and tumor infiltrating lymphocytes (TILs) testing. Forty-seven percent had P-L1 staining $>1 \%$ and $53 \%$ had evidence of TILs $>2+(>10$ TILs/HPF). At the time of data cut-off, 16 patients were evaluable for response. ORR was $4.7 \%$, with one PR and no CRs. Of the remaining patients, 3 (4.7\%) patients experienced SD and $12(57 \%)$ experienced PD. The one patient with a PR 


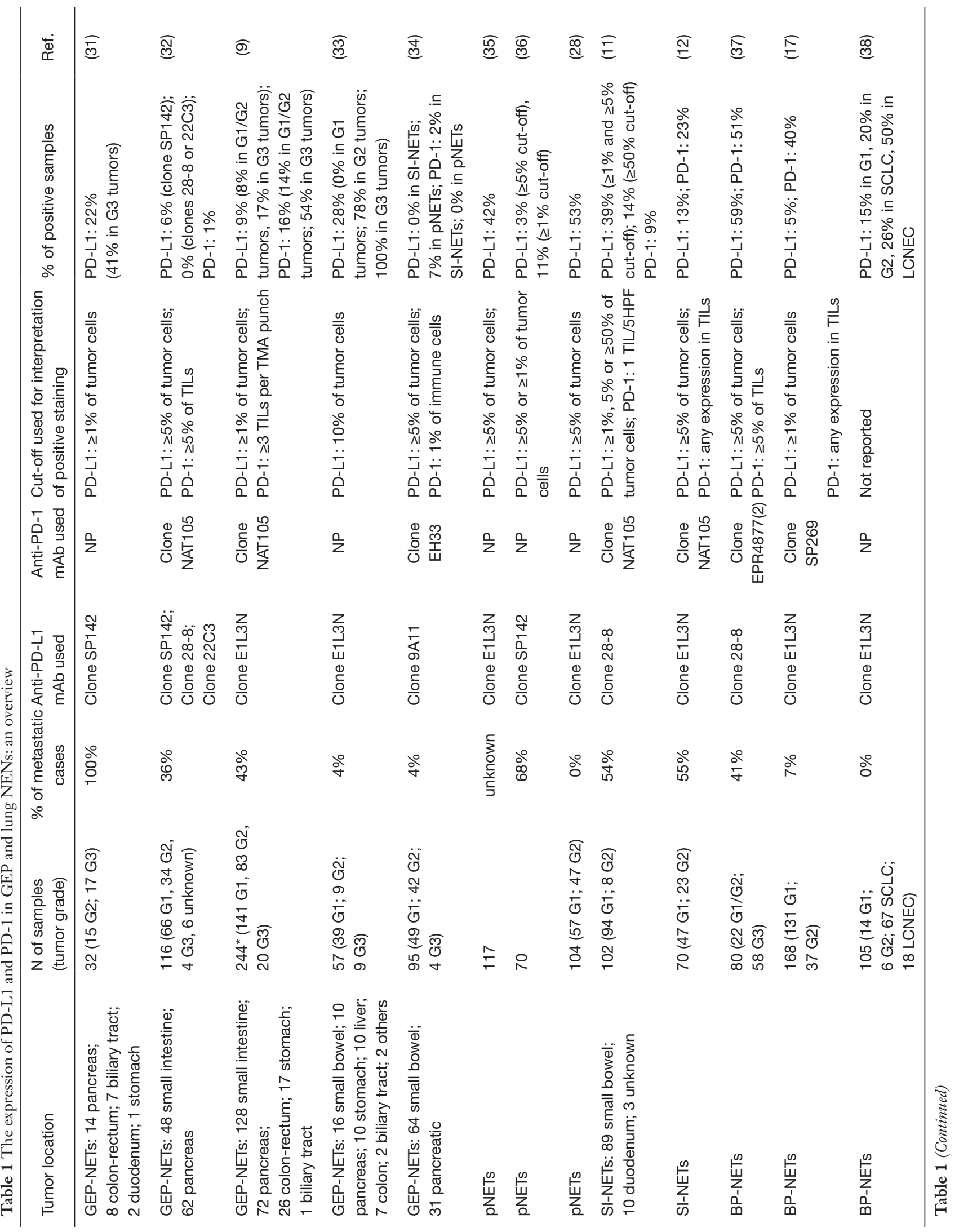




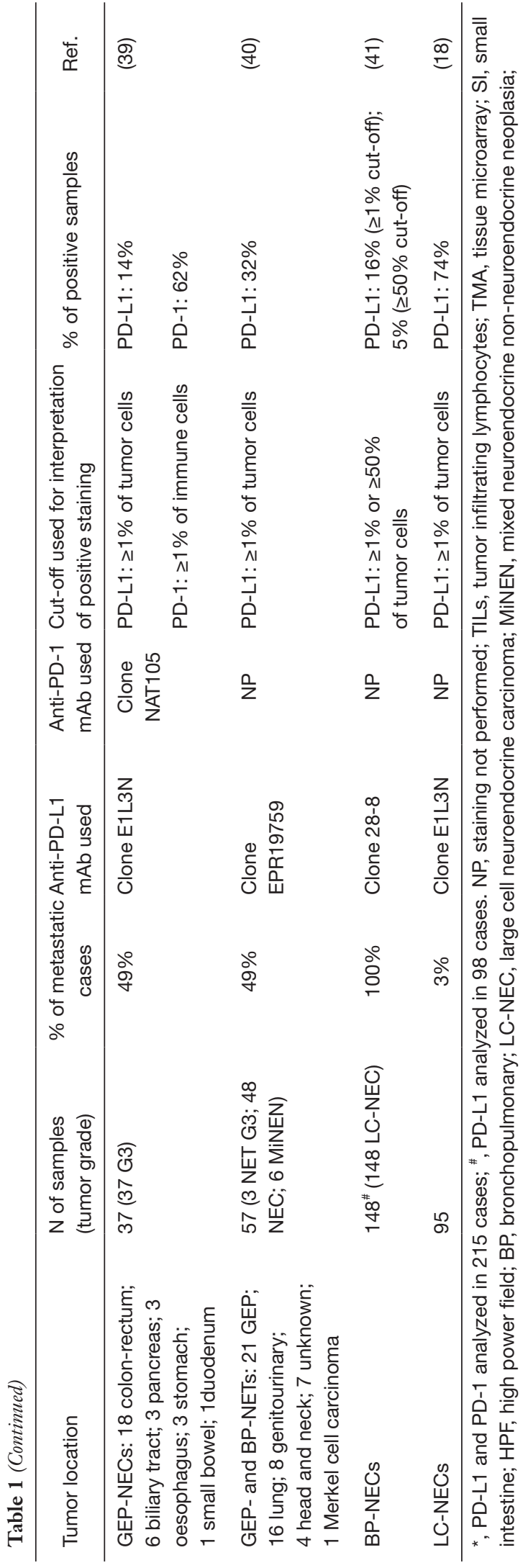

was negative for PD-L1 and had evidence of $>20$ TILs/ HPF. Median PFS was 9.14 weeks (95\% CI, 6.71-13.14 weeks) and median OS was 15.4 weeks (95\% CI, 13 weeksnot reached). Treatment-related AEs occurred in 37\% of patients, with $28 \%$ having grade 3 AEs.

Two similar phase II studies were conducted in patients with high-grade NENs who had progressed on prior platinum-based therapy, one utilizing avelumab in NENs of any primary origin excluding small cell lung cancer and Merkel cell carcinoma, and another with pembrolizumab in extrapulmonary NECs (excluding well-differentiated grade 3 NENs) $(45,46)$. Twenty-nine patients were treated with avelumab, and at time of data cut-off, median DCR after 8 weeks of treatment was $32 \%$ with 2 PRs (7\%), and median OS was 4.2 months. Treatment related AEs occurred in $38 \%$ of patients, with $4 \%$ having grade 3 AEs. The pembrolizumab trial was designed as a 2-stage study, with patients enrolled on stage 1 receiving pembrolizumab monotherapy. Data from stage 1 of the study was recently presented, reporting that of 14 patients enrolled, ORR was $7 \%$. Median PFS was 58 days and $43 \%$ of patients discontinued treatment for clinical deterioration or radiographic $\mathrm{PD}$ prior to the first scheduled scan at 9 weeks. At last follow-up, one patient was still on treatment after 19 cycles. Treatment related AEs were mild, with no patients experiencing grade 3-5 AEs attributable to therapy.

A phase II study of spartalizumab (PDR001), a humanized anti-PD-1 antibody, was conducted in patients with nonfunctional, well and poorly-differentiated NENs (47). Patients with a well-differentiated NET of GEP or thoracic origin, refractory to prior anti-cancer therapies, including everolimus, or poorly differentiated GEP NEC patients who progressed on at least one prior line of cytotoxic chemotherapy were eligible for enrollment. Patients were enrolled regardless of PD-L1 expression. The primary endpoint was ORR, assessed per RECIST 1.1 by independent central radiology review. ORR was $7.4 \%$ in well-differentiated NETs and $4.8 \%$ in poorlydifferentiated GEP NECs. Patients with lung NETs had a higher ORR at $20 \%$, although 2 of 6 responding patients expired shortly after initial response. Among patients with poorly-differentiated GEP-NECs, welldifferentiated GEP-NETs and lung NETs, the rate of expression of PD-L1 in immune cells was $42 \%, 23 \%$ and $15 \%$ respectively. Biomarker results suggested a potential link between TIM-3 expression and lack of treatment response. 


\section{Combination IO therapy trials}

The Southwest Oncology Group (SWOG) DART (Dual Anti-CTLA-4 and Anti-PD-1 Blockade in Rare Tumors) trial is a phase II basket trial of ipilimumab and nivolumab in rare tumors, including two cohorts of NENs: one defined as "neuroendocrine carcinoma, including carcinoid of the lung" but enrolling well and poorly differentiated NENs of any primary site (48). and the other defined as "endocrine carcinoma of the pancreas and digestive tract", but enrolling well and poorly differentiated NENs. Patients are eligible if they progressed on at least one prior line of therapy. Study treatment consists of ipilimumab $1 \mathrm{mg} / \mathrm{kg}$ every 6 weeks and nivolumab $240 \mathrm{mg}$ every 2 weeks until local investigator determined progression of disease. Preliminary analysis of the "neuroendocrine carcinoma including carcinoid of the lung" cohort was recently presented. Fifty-eight percent of patients had high-grade cancers (differentiation not well defined), $30 \%$ had intermediate-grade tumors, and $12 \%$ low-grade tumors. The ORR was $24 \%$, all responders with high-grade tumors (which included 2 high-grade lung NECs). Forty-two percent of high-grade tumors responded while none of the low or intermediate grade tumors responded. Six-month PFS was $30 \%$ and mean OS was 11 months at the time of data cut-off. The toxicity profile was relatively mild, with $30 \%$ of patients reporting fatigue and $27 \%$ reporting nausea. Elevated alanine aminotransferase (ALT) was the most common (9\%) grade 3-4 immunerelated $\mathrm{AE}$.

\section{Towards novel immunotherapeutic strategies}

In addition to checkpoint inhibitors, bispecific tumortargeting antibodies (BsAbs) are a new class of drugs allowing for simultaneous engagement of two targets, theoretically increasing binding specificity, allowing for dual activation or blockade of two disease mediators. A recent, phase I, first-in-human study of a new BsAb (XmAb18087) targeting somatostatin receptor (SSTR) subtype 2 and CD3 in welldifferentiated neuroendocrine and gastrointestinal stromal (GIST) tumors began accrual in early 2018 and recruitment is ongoing (NCT03411915).

Adoptive transfer of genetically-modified autologous $\mathrm{T}$ cells is gaining traction as one of the most promising advances in cancer immunotherapy, and impressive outcomes have been recently recorded in clinical trials of chimeric antigen receptor (CAR) $\mathrm{T}$ cells targeting CD19 or $\mathrm{B}$ cell maturation antigen (BCMA) in patients with $\mathrm{B}$ cell malignancies (49). CARs are synthetic fusion proteins consisting of an extracellular antigen-recognition domain linked to an intracellular activating domain. Once activated, CAR T cells proliferate and exert their effector functions including lysis of target cells, leading to "epitope spreading" and consequent induction of a secondary immune response against the tumor. Research is currently underway to develop CAR T cells directed against somatostatin receptorexpressing NET cells. Data presented this year show preliminary evidence of antitumor activity against NET cell lines and experiments in mice are currently underway (50).

Oncolytic viruses engineered to selectively kill tumor cells have exhibited activity in melanoma and head and neck cancers (51-53). An oncolytic adenovirus (AdVince) for the treatment of liver metastases from NETs was recently developed and is now being evaluated in a phase I/ IIa clinical trial for patients with liver dominant NETs of GEP or bronchial origin (NCT02749331). The adenovirus is designed to utilize the gene promoter from human chromogranin A for selective replication in neuroendocrine cells, and in preclinical evaluation of the virus, was found to successfully replicate in and kill NET cells without inducing a considerable amount of proinflammatory cytokines or chemokines in blood (54).

\section{Conclusions}

While the majority of well-differentiated NETs are "immunologically cold," poorly differentiated NECs are more likely to express PD-L1 in the presence of an abundant $\mathrm{T}$ cell infiltration. Single agent PD-1 inhibitor therapy has demonstrated limited activity in well-differentiated NETs, although preliminary evidence suggests that lung NETs may be mildly more immunosensitive than NETs of the GI tract. Surprisingly, PD-1 inhibitors have also shown limited activity in patients with poorly differentiated NECs (excluding Merkel Cell Cancers and Lung NECs, which are biologically distinct). Very early data suggest that combination ipilimumab/nivolumab treatment may be associated with promising activity in poorly differentiated NECs. These preliminary findings require confirmation. Novel immunotherapeutic approaches such as bispecific antibodies and CAR-T cells may one day represent a new paradigm for the treatment of well-differentiated NETs.

\section{Acknowledgments}

None. 


\section{Footnote}

Conflicts of Interest: J Strosberg: Consult (Novartis); Speakers bureau (Ipsen and Lexicon). The other authors have no conflicts of interest to declare.

Ethical Statement: The authors are accountable for all aspects of the work in ensuring that questions related to the accuracy or integrity of any part of the work are appropriately investigated and resolved.

\section{References}

1. Cives M, Strosberg JR. Gastroenteropancreatic Neuroendocrine Tumors. CA Cancer J Clin 2018;68:471-87.

2. Scarpa A, Chang DK, Nones K, et al. Whole-genome landscape of pancreatic neuroendocrine tumours. Nature 2017;543:65-71.

3. Fernandez-Cuesta L, Peifer M, Lu X, et al. Frequent mutations in chromatin-remodelling genes in pulmonary carcinoids. Nat Commun 2014;5:3518.

4. Waddell N, Pajic M, Patch AM, et al. Whole genomes redefine the mutational landscape of pancreatic cancer. Nature 2015;518:495-501.

5. Cives M, Pelle E, Quaresmini D, et al. The Tumor Microenvironment in Neuroendocrine Tumors: Biology and Therapeutic Implications. Neuroendocrinology 2019;109:83-99.

6. Lawrence MS, Stojanov P, Polak P, et al. Mutational heterogeneity in cancer and the search for new cancerassociated genes. Nature 2013;499:214-8.

7. Vijayvergia N, Boland PM, Handorf E, et al. Molecular profiling of neuroendocrine malignancies to identify prognostic and therapeutic markers: a Fox Chase Cancer Center Pilot Study. Br J Cancer 2016;115:564-70.

8. Katz SC, Donkor C, Glasgow K, et al. T cell infiltrate and outcome following resection of intermediate-grade primary neuroendocrine tumours and liver metastases. HPB (Oxford) 2010;12:674-83.

9. Bösch F, Bruwer K, Altendorf-Hofmann A, et al. Immune checkpoint markers in gastroenteropancreatic neuroendocrine neoplasia. Endocr Relat Cancer 2019;26:293-301.

10. Takahashi D, Kojima M, Suzuki T, et al. Profiling the Tumour Immune Microenvironment in Pancreatic Neuroendocrine Neoplasms with Multispectral Imaging Indicates Distinct Subpopulation Characteristics Concordant with WHO 2017 Classification. Sci Rep
2018;8:13166.

11. Cives M, Strosberg J, Al Diffalha S, et al. Analysis of the immune landscape of small bowel neuroendocrine tumors. Endocr Relat Cancer 2019;26:119-30.

12. Lamarca A, Nonaka D, Breitwieser W, et al. PD-L1 expression and presence of TILs in small intestinal neuroendocrine tumours. Oncotarget 2018;9:14922-38.

13. Vikman S, Giandomenico V, Sommaggio R, et al. CD8+ $\mathrm{T}$ cells against multiple tumor-associated antigens in peripheral blood of midgut carcinoid patients. Cancer Immunol Immunother 2008;57:399-409.

14. Koşaloğlu Z, Zornig I, Halama N, et al. Identification of immunotherapeutic targets by genomic profiling of rectal NET metastases. Oncoimmunology 2016;5:e1213931.

15. Kasajima A, Ishikawa $Y$, Iwata A, et al. Inflammation and PD-L1 expression in pulmonary neuroendocrine tumors. Endocr Relat Cancer 2018;25:339-50.

16. Wang H, Li Z, Dong B, et al. Prognostic significance of PD-L1 expression and CD8+ T cell infiltration in pulmonary neuroendocrine tumors. Diagn Pathol 2018;13:30.

17. Vesterinen T, Kuopio T, Ahtiainen M, et al. PD-1 and PD-L1 expression in pulmonary carcinoid tumors and their association to tumor spread. Endocr Connect 2019;8:1168-75.

18. Ohtaki Y, Kaira K, Atsumi J, et al. Prognostic significance of PD-L1 expression and tumor infiltrating lymphocytes in large cell neuroendocrine carcinoma of lung. Am J Transl Res 2018;10:3243-53.

19. de Reuver PR, Mehta S, Gill P, et al. Immunoregulatory Forkhead Box Protein p3-Positive Lymphocytes Are Associated with Overall Survival in Patients with Pancreatic Neuroendocrine Tumors. J Am Coll Surg 2016;222:281-7.

20. Vikman S, Sommaggio R, De La Torre M, et al. Midgut carcinoid patients display increased numbers of regulatory $T$ cells in peripheral blood with infiltration into tumor tissue. Acta Oncol 2009;48:391-400.

21. Funa K, Alm GV, Ronnblom L, et al. Evaluation of the natural killer cell-interferon system in patients with midgut carcinoid tumours treated with leucocyte interferon. Clin Exp Immunol 1983;53:716-24.

22. Aparicio-Pagés MN, Verspaget HW, Pena AS, et al. Natural killer cell activity in patients with neuroendocrine tumours of the gastrointestinal tract; relation with circulating gastrointestinal hormones. Neuropeptides 1991;20:1-7.

23. Soucek L, Buggy JJ, Kortlever R, et al. Modeling 
pharmacological inhibition of mast cell degranulation as a therapy for insulinoma. Neoplasia 2011;13:1093-100.

24. Soucek L, Lawlor ER, Soto D, et al. Mast cells are required for angiogenesis and macroscopic expansion of Myc-induced pancreatic islet tumors. Nat Med 2007;13:1211-8.

25. Pyonteck SM, Gadea BB, Wang HW, et al. Deficiency of the macrophage growth factor CSF-1 disrupts pancreatic neuroendocrine tumor development. Oncogene 2012;31:1459-67.

26. Krug S, Abbassi R, Griesmann H, et al. Therapeutic targeting of tumor-associated macrophages in pancreatic neuroendocrine tumors. Int J Cancer 2018;143:1806-16.

27. Wei IH, Harmon CM, Arcerito M, et al. Tumor-associated macrophages are a useful biomarker to predict recurrence after surgical resection of nonfunctional pancreatic neuroendocrine tumors. Ann Surg 2014;260:1088-94.

28. Cai L, Michelakos T, Deshpande V, et al. Role of TumorAssociated Macrophages in the Clinical Course of Pancreatic Neuroendocrine Tumors (PanNETs). Clin Cancer Res 2019;25:2644-55.

29. Katsenelson NS, Shurin GV, Bykovskaia SN, et al. Human small cell lung carcinoma and carcinoid tumor regulate dendritic cell maturation and function. Mod Pathol 2001;14:40-5.

30. Ryschich E, Autschbach F, Eisold S, et al. Expression of HLA class I/II antigens and $\mathrm{T}$ cell immune response in human neuroendocrine tumors of the pancreas. Tissue Antigens 2003;62:48-54.

31. Kim ST, Ha SY, Lee S, et al. The Impact of PD-L1 Expression in Patients with Metastatic GEP-NETs. J Cancer 2016;7:484-9.

32. Sampedro-Núñez M, Serrano-Somavilla A, Adrados $\mathrm{M}$, et al. Analysis of expression of the PD-1/PD-L1 immune checkpoint system and its prognostic impact in gastroenteropancreatic neuroendocrine tumors. Sci Rep 2018;8:17812.

33. Cavalcanti E, Armentano R, Valentini AM, et al. Role of PD-L1 expression as a biomarker for GEP neuroendocrine neoplasm grading. Cell Death Dis 2017;8:e3004.

34. da Silva A, Bowden M, Zhang S, et al. Characterization of the Neuroendocrine Tumor Immune Microenvironment. Pancreas 2018;47:1123-9.

35. Fan $\mathrm{Y}, \mathrm{Ma} \mathrm{K}$, Wang $\mathrm{C}$, et al. Prognostic value of $\mathrm{PD}-$ $\mathrm{L} 1$ and PD-1 expression in pulmonary neuroendocrine tumors. Onco Targets Ther 2016;9:6075-82.

36. Ferrata M, Schad A, Zimmer S, et al. PD-L1 Expression and Immune Cell Infiltration in Gastroenteropancreatic
(GEP) and Non-GEP Neuroendocrine Neoplasms With High Proliferative Activity. Front Oncol 2019;9:343.

37. Roberts JA, Gonzalez RS, Das S, et al. Expression of PD-1 and PD-L1 in poorly differentiated neuroendocrine carcinomas of the digestive system: a potential target for anti-PD-1/PD-L1 therapy. Hum Pathol 2017;70:49-54.

38. Sagnas C, Blank A, Franzelli M, et al. PD-L1 expressed in a subset of pancreatic neuroendocrine tumors (pNET). Paper presented at: 14th Annual ENETS Conference2017; Barcelona, Spain.

39. Hermans BCM, Derks JL, Thunnissen E, et al. Prevalence and prognostic value of PD-L1 expression in molecular subtypes of metastatic large cell neuroendocrine carcinoma (LCNEC). Lung Cancer 2019;130:179-86.

40. Ichiki Y, Matsumiya H, Mori M, et al. Predictive factors of postoperative survival among patients with pulmonary neuroendocrine tumor. J Thorac Dis 2018;10:6912-20.

41. Yang MW, Fu XL, Jiang YS, et al. Clinical significance of programmed death 1/programmed death ligand 1 pathway in gastric neuroendocrine carcinomas. World J Gastroenterol 2019;25:1684-96.

42. Mehnert JM, Rugo HS, O'Neil BH, et al. Pembrolizumab for patients with PD-L1-positive advanced carcinoid or pancreatic neuroendocrine tumors: results from the KEYNOTE-028 study. Paper presented at: ESMO 2017 Congress 2017; Barcelona, Spain.

43. Ennishi D, Jiang A, Boyle M, et al. Double-Hit Gene Expression Signature Defines a Distinct Subgroup of Germinal Center B-Cell-Like Diffuse Large B-Cell Lymphoma. J Clin Oncol 2019;37:190-201.

44. Vijayvergia N, Dasari A, Ross EA, et al. Pembrolizumab (P) monotherapy in patients with previously treated metastatic high grade neuroendocrine neoplasms (HG-NENs). J Clin Oncol 2018;36:4104.

45. Fottner C, Apostolidis L, Ferrata M, et al. A phase II, open label, multicenter trial of avelumab in patients with advanced, metastatic high-grade neuroendocrine carcinomas NEC G3 (WHO 2010) progressive after first-line chemotherapy (AVENEC). J Clin Oncol 2019;37:4103.

46. Mehta RS, Rezvani K, Shpall EJ. Cord Blood Expansion: A Clinical Advance. J Clin Oncol 2019;37:363-6.

47. Yao JC, Strosberg J, Fazio N, et al. 1308OActivity \& safety of spartalizumab (PDR001) in patients (pts) with advanced neuroendocrine tumors (NET) of pancreatic (Pan), gastrointestinal (GI), or thoracic (T) origin, \&amp; gastroenteropancreatic neuroendocrine carcinoma (GEP NEC) who have progressed on prior treatment (Tx). Ann 
Oncol 2018. doi: 10.1093/annonc/mdy293.

48. Patel SP, Othus M, Chae YK, et al. Abstract CT039: A Phase II basket trial of dual anti-CTLA-4 and antiPD-1 blockade in rare tumors (DART) S1609: The neuroendocrine cohort. Cancer Res 2019;79:CT039.

49. June CH, O'Connor RS, Kawalekar OU, et al. CAR T cell immunotherapy for human cancer. Science 2018;359:1361-5.

50. Mandriani B, Cives M, Pelle E, et al. Development of Anti-SSTR CAR-T Cells for Future Treatment of NETs. Paper presented at: NANETS2019; Boston, MA.

51. Grigg C, Blake Z, Gartrell R, et al. Talimogene laherparepvec (T-Vec) for the treatment of melanoma and other cancers. Semin Oncol 2016;43:638-46.

doi: $10.21037 / \operatorname{tgh} .2019 .12 .18$

Cite this article as: Al-Toubah T, Cives M, Strosberg J. Novel immunotherapy strategies for treatment of neuroendocrine neoplasms. Transl Gastroenterol Hepatol 2020;5:54.
52. Andtbacka RH, Kaufman HL, Collichio F, et al. Talimogene Laherparepvec Improves Durable Response Rate in Patients With Advanced Melanoma. J Clin Oncol 2015;33:2780-8.

53. Mell LK, Brumund KT, Daniels GA, et al. Phase I Trial of Intravenous Oncolytic Vaccinia Virus (GLONC1) with Cisplatin and Radiotherapy in Patients with Locoregionally Advanced Head and Neck Carcinoma. Clin Cancer Res 2017;23:5696-702.

54. Yu D, Leja-Jarblad J, Loskog A, et al. Preclinical Evaluation of AdVince, an Oncolytic Adenovirus Adapted for Treatment of Liver Metastases from Neuroendocrine Cancer. Neuroendocrinology 2017;105:54-66. 